\title{
Assessment of Testing Methods for Drug-Induced Repolarization Delay and Arrhythmias in an iPS Cell-Derived Cardiomyocyte Sheet: Multi-site Validation Study
}

\author{
Yuji Nakamura ${ }^{1}$, Junko Matsuo ${ }^{1,2,3}$, Norimasa Miyamoto ${ }^{4}$, Atsuko Ojima ${ }^{4}$, Kentaro Ando ${ }^{1}$, Yasunari Kanda ${ }^{2}$, \\ Kohei Sawada $^{4}$, Atsushi Sugiyama ${ }^{1, * a}$, and Yuko Sekino ${ }^{2,5, * b}$ \\ ${ }^{1}$ Department of Pharmacology, Faculty of Medicine, Toho University, Tokyo 143-8540, Japan \\ ${ }^{2}$ Division of Pharmacology, National Institute of Health Sciences, Tokyo 158-8501, Japan \\ ${ }^{3}$ Drug Safety Research Laboratories, Shin Nippon Biomedical Laboratories, Ltd., Kagoshima 891-1394, Japan \\ ${ }^{4}$ Biopharmaceutical Assessments Core Function Unit, Eisai Product Creation Systems, Eisai Co., Ltd., Tsukuba 300-2635, Japan \\ ${ }^{5}$ Department of Neurobiology and Behavior, Gunma University Graduate School of Medicine, Gunma 371-8511, Japan
}

Received November 26, 2013; Accepted February 11, 2014

\begin{abstract}
A prospective comparison study across 3 independent research laboratories of a pure $\mathrm{I}_{\mathrm{Kr}}$ blocker E-4031 was conducted by using the same batch of human iPS cell-derived cardiomyocytes in order to verify the utility and reliability of our original standard protocol. Field potential waveforms were recorded with a multi-electrode array system to measure the inter-spike interval and field potential duration. The effects of E-4031 at concentrations of 1 to $100 \mathrm{nM}$ were sequentially examined every $10 \mathrm{~min}$. In each facility, E-4031 significantly prolonged the field potential duration corrected by Fridericia's formula and caused early afterdepolarizations occasionally resulting in triggered activities, whereas it tended to decrease the rate of spontaneous contraction. These results were qualitatively and quantitatively consistent with previous nonclinical in vitro and in vivo studies as well as clinical reports. There were inter-facility differences in some absolute values of the results, which were not observed when the values were normalized as percentage change. Information described in this paper may serve as a guide when predicting the drug-induced repolarization delay and arrhythmias with this new technology of stem cells.
\end{abstract}

Keywords: E-4031, iPS cell-derived cardiomyocyte, multi-site validation, field potential, TdP

\section{Introduction}

Drug-induced proarrhythmia has been a major safety concern about the development of new drugs, leading to issuing of ICH E14 and S7B guidelines in May 2005 $(1,2)$. The guidelines have effectively reduced risks of a new compound causing torsades de pointes, whereas non-clinical and clinical studies in the current approach still remain imperfect because they identify many drugs as being "positive" despite a lack of demonstrable proarrhythmic risk (3-6). In a recent workshop held in July 2013 by the US Food and Drug Administration (FDA),

Corresponding authors.

*aatsushi.sugiyama@med.toho-u.ac.jp,*byukos@nihs.go.jp

Published online in J-STAGE on April 2, 2014

doi: 10.1254/jphs.13248FP the Cardiac Safety Research Consortium and the nonprofit Health and Environmental Sciences Institute (HESI), a new paradigm was proposed and discussed, focusing on a comprehensive assessment of multi ion channel effects to determine actual proarrhythmic risk of drugs (7). This new approach will include a stem-cell technology that has the potential to improve the currently used assessment of cardiotoxicity; however, more work is required prior to the use of stem cell-derived cardiomyocyte models to accurately predict proarrhythmias in humans (7).

There have been a large number of various studies with stem cell-derived cardiomyocytes examining electrophysiological effects of drugs $(8-13)$. In an effort to further improve upon the assay system, this report describes a more simple and reliable protocol of an induced pluripotent stem (iPS) cell-derived, cardio- 
myocyte-sheet model. Extensive preliminary studies have confirmed that the protocol proposed in this paper could be optimal for assessing E-4031-induced repolarization delay and arrhythmias and would qualitatively and quantitatively reflects its electropharmacological profile in humans. This is a critically new finding and a significant improvement over the previous in vitro $\mathrm{I}_{\mathrm{Kr}}$ assay systems including the hERG potassium channels and the papillary muscle of guinea pigs. In this study, in order to start verifying the reproducibility of our protocol, a prospective comparison study of E-4031 was conducted across 3 independent research laboratories with the same batch of iPS cell-derived cardiomyocytes.

\section{Materials and Methods}

\section{Cell culture and plating}

Each facility (E, N, T) obtained the same batch (\#1089404) of cryopreserved human iPS cell-derived cardiomyocytes [iCells; Cellular Dynamics International (CDI), Madison, WI, USA]. The cells were thawed in specially prepared medium (Plating Media, CDI), which were plated onto $0.1 \%$ gelatin-coated, 6-well tissueculture plates (Becton Dickinson, Franklin Lakes, NJ, USA) at a density of $1.3-2.6 \times 10^{6}\left(\mathrm{E}: 1.3 \times 10^{6}, \mathrm{~N}\right.$ : $2.0 \times 10^{6}$, T: $2.4-2.6 \times 10^{6}$ ) of cells per well. Two days after plating, Plating Media was replaced to specially prepared culture medium (Maintenance Media, CDI). Then, the culture medium was changed with fresh one every 2 days. The cells were cultured for $3.7 \pm 1.4$ days (2- 7 days) after thawing at $37^{\circ} \mathrm{C}$ with $5 \% \mathrm{CO}_{2}$ prior to re-plating.

The electrical activity of cardiomyocytes was measured by using our original protocol. Briefly, the recording area of probes with 64 of the recording electrodes (MED probe; MED-P515A, Alpha Med Scientific, Osaka) of the MED64 System (Alpha Med Scientific) was coated with $2 \mu \mathrm{L}$ of fibronectin $(50 \mu \mathrm{g}$ in $1 \mathrm{~mL}$ of distilled water), which was incubated at $37^{\circ} \mathrm{C}$ for $\geq 1 \mathrm{~h}$. The cells cultured in the 6-well tissue-culture plates were dispersed with $0.25 \%$ trypsin-EDTA or TrypLE Select, which were re-plated onto the MED probes at a density of $3 \times 10^{4}$ cells in a $2 \mu \mathrm{L}$ of the culture medium. The cells were incubated at $37^{\circ} \mathrm{C}$ with $5 \% \mathrm{CO}_{2}$ for $2-18$ h (E: $4-12 \mathrm{~h}, \mathrm{~N}: 2-18 \mathrm{~h}, \mathrm{~T}: 12-18 \mathrm{~h})$ in moisture condition prior to filling each probe with $1 \mathrm{~mL}$ of the culture medium. The half volume or all of the culture medium of the probes was changed with the culture medium, which had been warmed to $37^{\circ} \mathrm{C}$, every 2 days thereafter. The cells were cultured for $5.2 \pm 1.6$ days (3-7 days) to obtain a sheet of cardiomyocytes with spontaneous and synchronous electrical automaticity.

\section{Field potentials (FPs) assay}

Maintenance Media was used as a culture medium throughout the experiment. Prior to the measurement of FPs, cardiomyocyte sheets were equilibrated for $\geq 30$ min in the $\mathrm{CO}_{2}$ incubator in 1 or $2 \mathrm{~mL}$ of fresh culture media. After equilibration, the probes were kept at $36^{\circ} \mathrm{C}-37^{\circ} \mathrm{C}$ with thermo-control systems and covered with a lid, through which aeration of $95 \% \mathrm{O}_{2} / 5 \% \mathrm{CO}_{2}$ gas was provided. FPs from spontaneously beating cardiomyocyte sheets were recorded and digitized at $20 \mathrm{kHz}$ by using the MED64 System. The stability and constancy of the waveforms, inter-spike interval, and field potential duration (FPD) were confirmed for $\geq 20$ min. FPD was defined as an interval from the initial sharp deflection to the peak of the dome (8). Using the information obtained in this observation period, we selected 3-6 electrodes, which would be suitable for continuous monitoring of the FP configuration consisting of spike and dome. After recording the basal control state, the effects of 1, 3, 10, 30, and $100 \mathrm{nM}$ of E-4031 were assessed by adding stock solution cumulatively to the culture medium to obtain target concentrations. The final concentration of DMSO was limited to be $<0.6 \%$, since DMSO at a concentration of $<0.6 \%$ has been reported to hardly affect any of the variables assessed in this study (8). At each concentration, the FP was recorded for $\geq 10 \mathrm{~min}$ and the last 30 beats were extracted as a dataset to analyze waveforms, inter-spike interval, and FPDs according to the previous report (8). The datasets of concentrations were excluded from the statistical analysis, when early afterdepolarization and/or triggered activity were observed. Early afterdepolarization was defined as deflection occurring at the plateau of the dome, and sharp deflection originating from early afterdepolarization was judged as a triggered activity. FPD was corrected with Fridericia's formula, which was defined as the primary method of correction in this study [FPDcF $=$ FPD $/(\text { inter-spike interval } / 1000)^{1 / 3}$ ] (14). The values of inter-spike interval and FPDcF from the last 30 waves at each concentration were averaged.

\section{Drugs and chemicals}

E-4031 was obtained from WAKO (Osaka) or synthetized at Eisai Co., Ltd. (Tsukuba). Gelatin was obtained from Sigma (St. Louis, MO, USA). Fibronectin was obtained from Becton Dickinson or Invitrogen (Carlsbad, CA, USA). Trypsin-EDTA and TrypLE Select were obtained from Invitrogen.

\section{Data analyses and statistical assessment}

In each experiment, one electrode that satisfied the following two conditions was chosen: 1) FP was recorded whole through the experiment, and 2) The amplitude of 
the dome was the largest. The data were expressed as the mean \pm S.E.M. The effects of the drug on interspike interval and FPDcF obtained in each facility were evaluated with the paired $t$-test or one-way repeatedmeasures analysis of variance (ANOVA) followed by Contrasts for mean values comparison between the baseline value $(0 \mathrm{nM})$ and others. Meanwhile, interfacility variability was assessed with one-way factorial ANOVA followed by Fisher's test or unpaired $t$-test. A $P$ value $<0.05$ was considered statistically significant.

\section{Results}

The effects of E-4031 in concentrations of $0,1,3,10$, 30 , and $100 \mathrm{nM}$ were examined in each facility, except that $1 \mathrm{nM}$ was not performed in facility $\mathrm{N}$. The number of preparations that can be used for the assessment of inter-spike interval, field-potential duration, and categorical analysis decreased due to the onset of early afterdepolarization and/or triggered activity as the concentration of drug increased.

\section{Inter-spike interval}

The effects of the drug on the inter-spike interval (ms) are summarized in Fig. 1 (upper panel). The baseline values $(0 \mathrm{nM})$ were $926 \pm 44 \mathrm{~ms}$ in facility E, 1,216 \pm 56 $\mathrm{ms}$ in facility $\mathrm{N}$, and $956 \pm 22 \mathrm{~ms}$ in facility $\mathrm{T}$. Interfacility difference was observed between $\mathrm{N}$ and $\mathrm{E}$ besides between $\mathrm{N}$ and $\mathrm{T}$, which was not detected between $\mathrm{E}$ and $\mathrm{T}$. No significant change from the respective baseline values was detected at 1,3 , or $10 \mathrm{nM}$ in $\mathrm{E}$ and $\mathrm{T}$ and at $3 \mathrm{nM}$ in $\mathrm{N}$. Inter-facility difference was observed at $3 \mathrm{nM}$ between $\mathrm{N}$ and $\mathrm{E}$ besides between $\mathrm{N}$ and $\mathrm{T}$, which was not detected at any concentration between $\mathrm{E}$ and $\mathrm{T}$. Meanwhile, the effects of the drug on the inter-spike interval (\%) are summarized in Fig. 1 (lower panel). The significant increase was observed at $3 \mathrm{nM}$ in $\mathrm{N}$, which was not detected at 1,3 , or $10 \mathrm{nM}$ in $\mathrm{E}$ or $\mathrm{T}$, although the similar trend was observed. Interfacility difference was not detected at any concentration.

\section{Prolongation of field-potential duration}

The effects of the drug on the FPDcF (ms) are summarized in Fig. 2 (upper panel) and typical tracings of field potential before and after the drug treatment are depicted in Fig. 3. The baseline values $(0 \mathrm{nM})$ were $430 \pm 12 \mathrm{~ms}$ in E, $443 \pm 5 \mathrm{~ms}$ in $\mathrm{N}$, and $320 \pm 15 \mathrm{~ms}$ in $\mathrm{T}$. Inter-facility difference was detected between $\mathrm{T}$ and $\mathrm{E}$ besides between $\mathrm{T}$ and $\mathrm{N}$, which was not detected between $\mathrm{E}$ and $\mathrm{N}$. FPDcF was prolonged at 3 and $10 \mathrm{nM}$ in $\mathrm{E}$ and at $10 \mathrm{nM}$ in $\mathrm{T}$, which tended to be prolonged at $3 \mathrm{nM}$ in $\mathrm{N}$ without statistical significance. Inter-facility difference was detected at 1,3 , and $10 \mathrm{nM}$ between $\mathrm{E}$

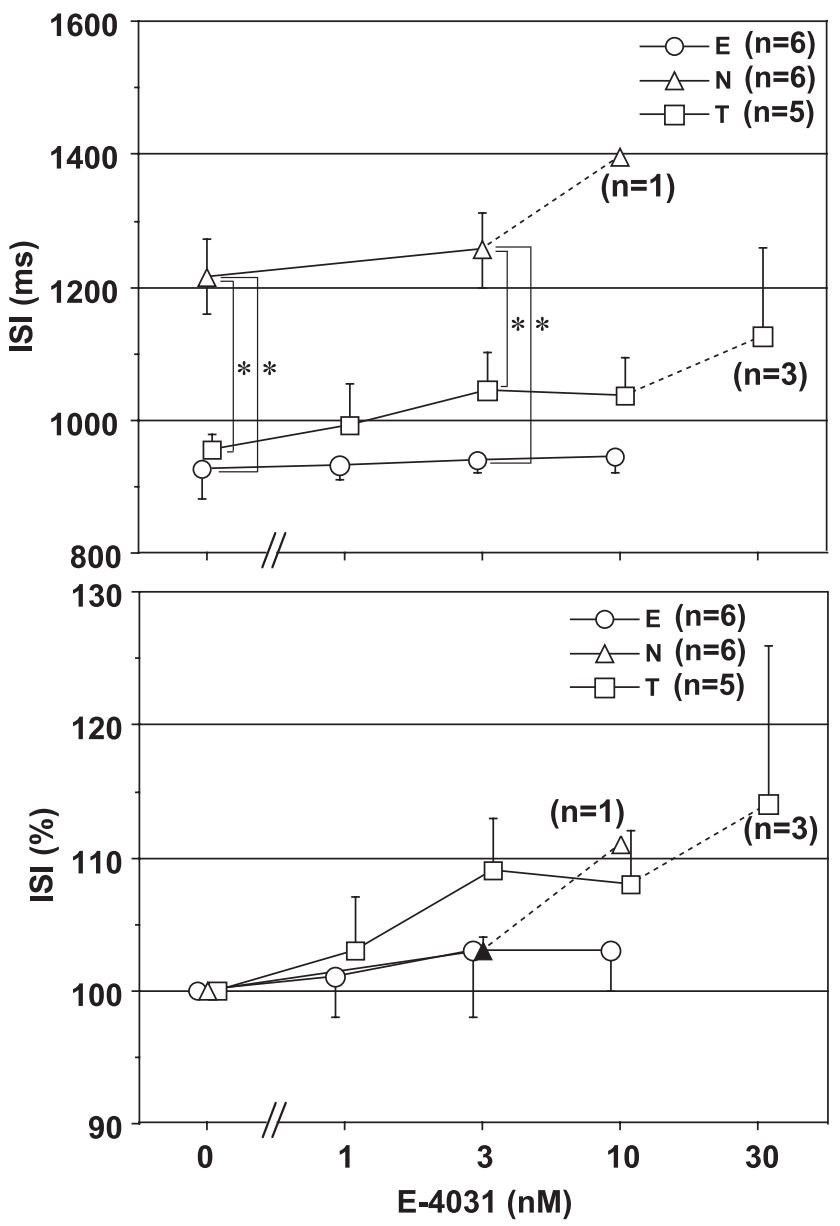

Fig. 1. Summary of the results showing the actual measurement values (upper) and their percentage changes (lower) in inter-spike interval (ISI) of E-4031 in human iPS cell-derived cardiomyocytes in each facility. Each value represents the mean \pm S.E.M. of 6 preparations for facility $\mathrm{E}$ and facility $\mathrm{N}$ and 5 preparations for facility $\mathrm{T}$. Values in parentheses represent the number of the preparations. An asterisk indicates significant difference between the facilities, whereas a closed symbol represents significant change from the respective baseline value.

and $\mathrm{T}$ and at $3 \mathrm{nM}$ between $\mathrm{N}$ and $\mathrm{T}$. Inter-facility difference was not detected at any concentration between $\mathrm{E}$ and N. Meanwhile, the effects of the drug on the FPDcF (\%) are summarized in Fig. 2 (lower panel). FPDcF was prolonged at 3 and $10 \mathrm{nM}$ in $\mathrm{E}$ and at $10 \mathrm{nM}$ in T, whereas it tended to be prolonged at $3 \mathrm{nM}$ in $\mathrm{N}$ without statistical significance. Inter-facility difference was not detected at any concentration.

Incidence of early afterdepolarization or triggered
activity
The incidence of early afterdepolarization or triggered
activity is summarized in Table 1 and typical tracing
of field potential with triggered activity is depicted in 


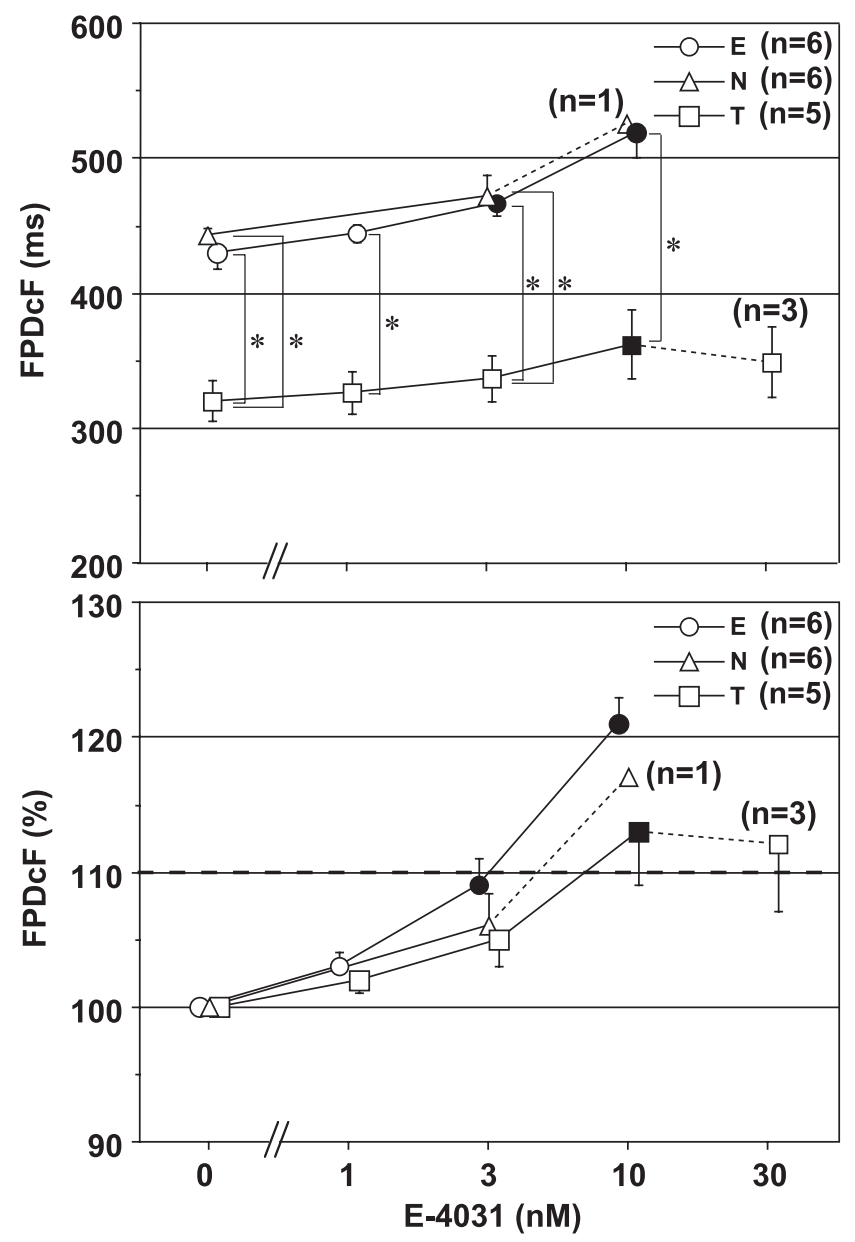

Fig. 2. Summary of the results showing the actual measurement values (upper) and their percentage changes (lower) in FPDcF of E-4031 in human iPS cell-derived cardiomyocytes in each facility. Each value represents the mean \pm S.E.M. of 6 preparations for facility $\mathrm{E}$ and facility $\mathrm{N}$ and 5 preparations for facility $\mathrm{T}$. Values in parentheses represent the number of the preparations. An asterisk indicates significant difference between facilities, whereas a closed symbol represents significant change from the respective baseline value.

Fig. 3. Early afterdepolarization or triggered activity was induced at $\geq 10 \mathrm{nM}$ in $\mathrm{N}$ and at $\geq 30 \mathrm{nM}$ in $\mathrm{E}$ and $\mathrm{T}$.

\section{Categorical analysis of FPDCF}

The results of categorical analysis of absolute FPDcF (ms) are summarized in Table 2. At the baseline and $1 \mathrm{nM}$, all FPDcF in each facility were categorized in $\leq 480 \mathrm{~ms}$, whereas FPDcF of $\geq 500 \mathrm{~ms}$ was observed at $\geq 3 \mathrm{nM}$ in $\mathrm{N}$ and at $\geq 10 \mathrm{nM}$ in $\mathrm{E}$, which was not observed in $\mathrm{T}$.

The results of categorical analysis of $\triangle \mathrm{FPDcF}$ are summarized in Table 3. At $1 \mathrm{nM}$, all $\triangle \mathrm{FPDcF}$ in $\mathrm{E}$ and $\mathrm{T}$ were categorized in $\leq 60 \mathrm{~ms}$. $\triangle \mathrm{FPDcF}$ of $>60 \mathrm{~ms}$ was observed at $\geq 3 \mathrm{nM}$ in $\mathrm{E}$ and $\mathrm{N}$ and at $\geq 10 \mathrm{nM}$ in $\mathrm{T}$.

\section{Discussion}

In this study, a prospective comparison study of E-4031 was conducted with the same batch of human iPS cell-derived cardiomyocytes in order to start verifying the reproducibility of our original standard protocol across 3 independent research laboratories. We demonstrated that the protocol can be reliable in detecting the drug-induced repolarization delay and arrhythmias with high reproducibility.

E-4031 tended to show a negative chronotropic effect at concentrations of $\geq 3 \mathrm{nM}$; however, a significant change was detected only at $3 \mathrm{nM}$ in facility $\mathrm{N}$ when assessed by percentage change (Fig. 1). A more potent negative chronotropic effect was observed by higher concentrations of E-4031 in each facility, although we did not perform the statistical analyses on inter-spike interval at concentrations of $\geq 10 \mathrm{nM}$ in facility $\mathrm{N}$ and $\geq 30 \mathrm{nM}$ in facilities $\mathrm{E}$ and $\mathrm{T}$ because of the limited number of experiments $(n=0-3)$. These results are in good accordance with a previous observation in patients with supraventricular tachyarrhythmias (15), in which E-4031 at a plasma concentration of $4.85 \pm 1.35 \mathrm{ng} / \mathrm{ml}$ $(11 \mathrm{nM})$ modestly prolonged RR interval, but it did not achieve statistical significance. Meanwhile in the single sinoatrial nodal cells of rabbits, E-4031 at a concentration of $100 \mathrm{nM}$ suppressed or blocked the spontaneous activity (16), and moreover in the Langendorff-perfused whole hearts of guinea pig, E-4031 at concentrations of $30-300 \mathrm{nM}$ or $5 \mu \mathrm{M}$ significantly reduced the heart rate $(17,18)$. Thus, our testing method using the human iPS cell-derived cardiomyocytes can be considered to be more sensitive than currently available in vitro nonclinical models in detecting the E-4031-induced negative chronotropic effect.

E-4031 caused early afterdepolarization and/or triggered activity in a concentration-related manner as shown in Fig. 3 and Table 1. In previous studies using the Langendorff-perfused rabbit heart, $0.5 \mu \mathrm{M}$ of E-4031 induced early afterdepolarization and triggered activity $(19,20)$. Also, in human embryonic stem cell-derived cardiomyocyte clusters, $1 \mu \mathrm{M}$ of E-4031 induced early afterdepolarization in half of the clusters (11). Meanwhile, in the human engineered heart tissue sheet made of the human embryonic stem cells, $10 \mathrm{nM}$ of E-4031 was reported to induce arrhythmias (12). Thus, our testing method as well as the previous human engineered heart tissue sheet is considered to have higher sensitivities than the human cardiomyocyte clusters or the Langendorff-perfused rabbit heart in detecting E-4031induced early afterdepolarization and/or triggered activity.

E-4031 prolonged the FPDcF in a concentrationrelated manner as shown in Figs. 2 and 3. A wide variety 


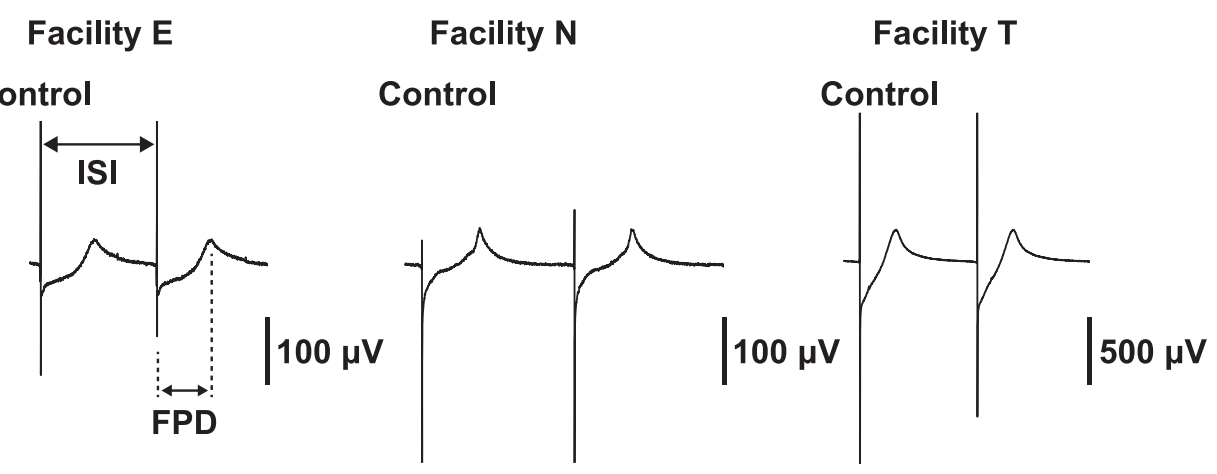

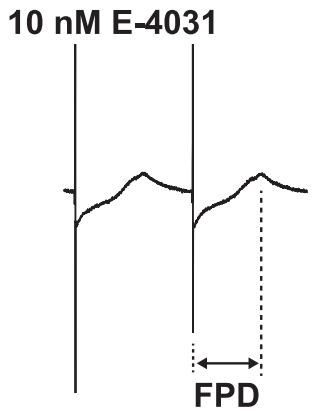

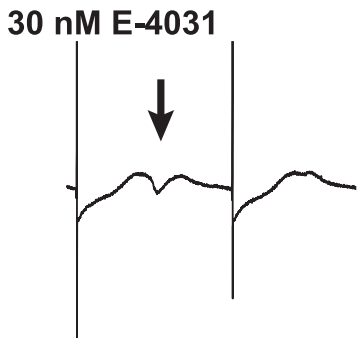

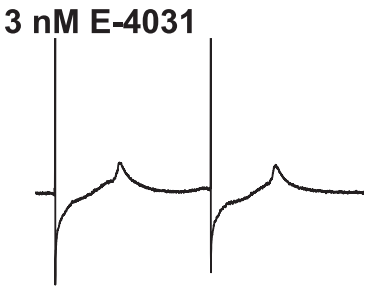

10 nM E-4031

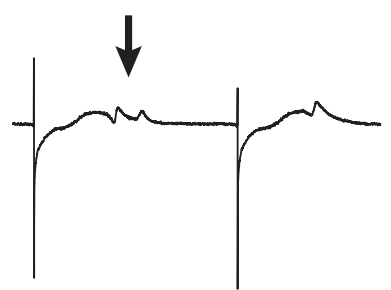

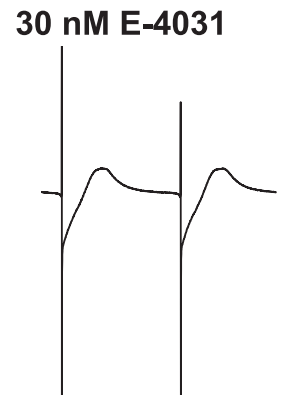

100 nM E-4031

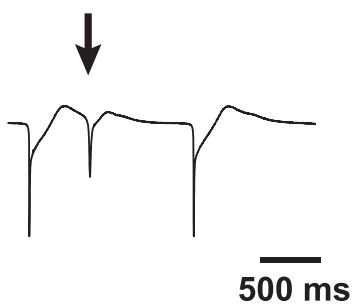

Fig. 3. Typical tracings of the field potential from each facility. Upper traces show the field potential at control (Control); middle traces indicate the prolongation of field potential duration (FPD) with 3-30 nM of E-4031; and lower traces represent the onset of early afterdepolarization or triggered activity with 10 - $100 \mathrm{nM} \mathrm{E-4031} \mathrm{(arrow).} \mathrm{ISI:} \mathrm{inter-spike} \mathrm{interval.}$

Table 1. Incidence of E-4031-induced early afterdepolarization or triggered activity

\begin{tabular}{|c|c|c|c|c|}
\hline \multirow{2}{*}{ Concentration (nM) } & \multicolumn{3}{|c|}{ Facility } & \multirow{2}{*}{ All } \\
\hline & $\mathrm{E}$ & $\mathrm{N}$ & $\mathrm{T}$ & \\
\hline 0 & $0 \%(0 / 6)$ & $0 \%(0 / 6)$ & $0 \%(0 / 5)$ & $0 \%(0 / 17)$ \\
\hline 1 & $0 \%(0 / 6)$ & NT & $0 \%(0 / 5)$ & $0 \%(0 / 11)$ \\
\hline 3 & $0 \%(0 / 6)$ & $0 \%(0 / 6)$ & $0 \%(0 / 5)$ & $0 \%(0 / 17)$ \\
\hline 10 & $0 \%(0 / 6)$ & $83 \%(5 / 6)$ & $0 \%(0 / 5)$ & $29 \%(5 / 17)$ \\
\hline 30 & $100 \%(6 / 6)$ & $100 \%(6 / 6)$ & $40 \%(2 / 5)$ & $82 \%(14 / 17)$ \\
\hline 100 & $100 \%(6 / 6)$ & $100 \%(6 / 6)$ & $100 \%(5 / 5)$ & $100 \%(17 / 17)$ \\
\hline
\end{tabular}

Note: The numerator in parentheses shows the number of preparations exerting early afterdepolarization or triggered activity, whereas the denominator indicates the total number of preparations assessed. NT: Not tested.

of analyses have been performed to clarify the effects of E-4031 on the repolarization process as summarized in Table $4(12,15,17-26)$. The effects of E-4031 in these previous reports are directionally the same as the currently observed results, although their potency varied greatly. Thus, the present findings suggest that the sensitivity of current testing method to detect drug-induced repolarization delay can be considered to be comparable to hERG assay and human subjects, but it may be higher than those of in vivo and in vitro animal models. 
Table 2. Summary of categorical analysis of absolute FPDcF values

\begin{tabular}{|c|c|c|c|c|c|}
\hline \multirow{2}{*}{$\begin{array}{c}\text { Concentration of E-4031 } \\
(\mathrm{nM})\end{array}$} & \multirow{2}{*}{$\begin{array}{c}\text { FPDcF } \\
(\mathrm{ms})\end{array}$} & \multicolumn{3}{|c|}{ Facility } & \multirow{2}{*}{ All } \\
\hline & & $\mathrm{E}$ & $\mathrm{N}$ & $\mathrm{T}$ & \\
\hline \multirow{4}{*}{0} & $\leq 450$ & $67 \%(4 / 6)$ & $83 \%(5 / 6)$ & $100 \%(5 / 5)$ & $82 \%(14 / 17)$ \\
\hline & $>450$ & $33 \%(2 / 6)$ & $17 \%(1 / 6)$ & $0 \%(0 / 5)$ & $18 \%(3 / 17)$ \\
\hline & $>480$ & $0 \%(0 / 6)$ & $0 \%(0 / 6)$ & $0 \%(0 / 5)$ & $0 \%(0 / 17)$ \\
\hline & $>500$ & $0 \%(0 / 6)$ & $0 \%(0 / 6)$ & $0 \%(0 / 5)$ & $0 \%(0 / 17)$ \\
\hline \multirow{4}{*}{1} & $\leq 450$ & $50 \%(3 / 6)$ & \multirow{4}{*}{ NT } & $100 \%(5 / 5)$ & $73 \%(8 / 11)$ \\
\hline & $>450$ & $50 \%(3 / 6)$ & & $0 \%(0 / 5)$ & $27 \%(3 / 11)$ \\
\hline & $>480$ & $0 \%(0 / 6)$ & & $0 \%(0 / 5)$ & $0 \%(0 / 11)$ \\
\hline & $>500$ & $0 \%(0 / 6)$ & & $0 \%(0 / 5)$ & $0 \%(0 / 11)$ \\
\hline \multirow{4}{*}{3} & $\leq 450$ & $17 \%(1 / 6)$ & $17 \%(1 / 6)$ & $100 \%(5 / 5)$ & $41 \%(7 / 17)$ \\
\hline & $>450$ & $50 \%(3 / 6)$ & $50 \%(3 / 6)$ & $0 \%(0 / 5)$ & $35 \%(6 / 17)$ \\
\hline & $>480$ & $33 \%(2 / 6)$ & $17 \%(1 / 6)$ & $0 \%(0 / 5)$ & $18 \%(3 / 17)$ \\
\hline & $>500$ & $0 \%(0 / 6)$ & $17 \%(1 / 6)$ & $0 \%(0 / 5)$ & $6 \%(1 / 17)$ \\
\hline \multirow{4}{*}{10} & $\leq 450$ & $17 \%(1 / 6)$ & $0 \%(0 / 1)$ & $80 \%(4 / 5)$ & $42 \%(5 / 12)$ \\
\hline & $>450$ & $0 \%(0 / 6)$ & $0 \%(0 / 1)$ & $20 \%(1 / 5)$ & $8 \%(1 / 12)$ \\
\hline & $>480$ & $17 \%(1 / 6)$ & $0 \%(0 / 1)$ & $0 \%(0 / 5)$ & $8 \%(1 / 12)$ \\
\hline & $>500$ & $67 \%(4 / 6)$ & $100 \%(1 / 1)$ & $0 \%(0 / 5)$ & $42 \%(5 / 12)$ \\
\hline \multirow{4}{*}{30} & $\leq 450$ & $-(0 / 0)$ & $-(0 / 0)$ & $100 \%(3 / 3)$ & $100 \%(3 / 3)$ \\
\hline & $>450$ & $-(0 / 0)$ & $-(0 / 0)$ & $0 \%(0 / 3)$ & $0 \%(0 / 3)$ \\
\hline & $>480$ & $-(0 / 0)$ & $-(0 / 0)$ & $0 \%(0 / 3)$ & $0 \%(0 / 3)$ \\
\hline & $>500$ & $-(0 / 0)$ & $-(0 / 0)$ & $0 \%(0 / 3)$ & $0 \%(0 / 3)$ \\
\hline
\end{tabular}

Note: The numerator in parentheses shows the number of preparations exerting respective FPDcF values in each category, whereas the denominator indicates the total number of preparations assessed. FPDcF $=$ FPD $/(\text { inter-spike interval } / 1000)^{1 / 3}$. NT: Not tested.

Table 3. Summary of categorical analysis of $\triangle \mathrm{FPDcF}$ values

\begin{tabular}{|c|c|c|c|c|c|}
\hline \multirow{2}{*}{$\begin{array}{l}\text { Concentration of E-4031 } \\
\text { (nM) }\end{array}$} & \multirow{2}{*}{$\triangle \mathrm{FPDcF}(\mathrm{ms})$} & \multicolumn{3}{|c|}{ Facility } & \multirow{2}{*}{ All } \\
\hline & & $\mathrm{E}$ & $\mathrm{N}$ & $\mathrm{T}$ & \\
\hline \multirow{3}{*}{1} & $\leq 30$ & $83 \%(5 / 6)$ & \multirow{3}{*}{ NT } & $100 \%(5 / 5)$ & $91 \%(10 / 11)$ \\
\hline & $>30$ & $17 \%(1 / 6)$ & & $0 \%(0 / 5)$ & $9 \%(1 / 11)$ \\
\hline & $>60$ & $0 \%(0 / 6)$ & & $0 \%(0 / 5)$ & $0 \%(0 / 11)$ \\
\hline \multirow{3}{*}{3} & $\leq 30$ & $67 \%(4 / 6)$ & $67 \%(4 / 6)$ & $100 \%(5 / 5)$ & $76 \%(13 / 17)$ \\
\hline & $>30$ & $0 \%(0 / 6)$ & $17 \%(1 / 6)$ & $0 \%(0 / 5)$ & $6 \%(1 / 17)$ \\
\hline & $>60$ & $33 \%(2 / 6)$ & $17 \%(1 / 6)$ & $0 \%(0 / 5)$ & $18 \%(3 / 17)$ \\
\hline \multirow{3}{*}{10} & $\leq 30$ & $0 \%(0 / 6)$ & $0 \%(0 / 1)$ & $60 \%(3 / 5)$ & $25 \%(3 / 12)$ \\
\hline & $>30$ & $17 \%(1 / 6)$ & $0 \%(0 / 1)$ & $20 \%(1 / 5)$ & $17 \%(2 / 12)$ \\
\hline & $>60$ & $83 \%(5 / 6)$ & $100 \%(1 / 1)$ & $20 \%(1 / 5)$ & $58 \%(7 / 12)$ \\
\hline \multirow{3}{*}{30} & $\leq 30$ & $-(0 / 0)$ & $-(0 / 0)$ & $33 \%(1 / 3)$ & $33 \%(1 / 3)$ \\
\hline & $>30$ & $-(0 / 0)$ & $-(0 / 0)$ & $33 \%(1 / 3)$ & $33 \%(1 / 3)$ \\
\hline & $>60$ & $-(0 / 0)$ & $-(0 / 0)$ & $33 \%(1 / 3)$ & $33 \%(1 / 3)$ \\
\hline
\end{tabular}

Note: The numerator in parentheses shows the number of preparations exerting respective $\triangle \mathrm{FPDcF}$ values in each category, whereas the denominator indicates the total number of preparations assessed. $\triangle$ FPDcF: Increase from baseline in FPDcF. NT: Not tested.

Since $82 \%$ of the basal FPDcF was $\leq 450 \mathrm{~ms}$, which is the upper limit of the normal range of QTc in human subjects, we examined the repolarization delays with the categorical analysis described in the ICH E14 guideline. $\mathrm{FPDcF}>500 \mathrm{~ms}$ and/or $\triangle \mathrm{FPDcF}>60 \mathrm{~ms}$ were detected at concentrations of $\geq 3 \mathrm{nM}$ in some preparations (Tables 2 and 3), indicating that $3 \mathrm{nM}$ of E-4031 will be a critical concentration for inducing the excessive QT-interval prolongation by this testing method.

The major purpose of this study was to clarify the 
Table 4. Summary of nonclinical and clinical reports regarding the effects of E-4031 on the repolarization markers

\begin{tabular}{|c|c|c|c|c|c|}
\hline Model & Method & Marker & Change $(\%)$ & Concentration (nM) & References \\
\hline hERG & & $\mathrm{IC}_{50}$ & & 7.7 & 21 \\
\hline \multirow[t]{8}{*}{ Guinea pig } & Ventricular myocytes & $\mathrm{APD}_{90}$ & 26 & 5,000 & 22 \\
\hline & & $\mathrm{APD}_{90}$ & 71 & 100 & 23 \\
\hline & Papillary muscles & $\mathrm{APD}_{70}$ & $9-68$ & $30-300$ & 23 \\
\hline & & $\mathrm{APD}_{90}$ & 10 & 20 & 24 \\
\hline & & $\mathrm{APD}_{30-90}$ & 10 & 7 & 24 \\
\hline & Langendorff heart & QTc & $5-27$ & $3-300$ & 18 \\
\hline & & $\mathrm{MAP}_{90}$ & $3-18$ & $3-300$ & 18 \\
\hline & & QTc & 26 & 5,000 & 17 \\
\hline \multirow[t]{2}{*}{ Rabbit } & Langendorff heart & QT & 51 & 500 & 19 \\
\hline & & $\mathrm{MAP}_{90}$ & 50 & 500 & 19 \\
\hline \multirow[t]{3}{*}{ Dog } & In vivo (Anesthetized) & QT & 57 & 6.2 & 25 \\
\hline & & QTc & 10 & 5.1 & 24 \\
\hline & In vivo (Conscious) & QTc & 10 & 19.2 & 24 \\
\hline Monkey & In vivo (Conscious) & QTc & 10 & 3.1 & 24 \\
\hline \multirow[t]{2}{*}{ Human } & & QTc & 14 & 12.1 & 15 \\
\hline & & QTc & $4.7-15$ & $5.1-27$ & 26 \\
\hline \multirow[t]{3}{*}{ iPS cell } & Single cell & $\mathrm{APD}_{90}$ & $40-70$ & $30-100$ & 20 \\
\hline & & $\mathrm{APD}_{90}$ & $5-11$ & $10-1,000$ & 12 \\
\hline & & $\mathrm{APD}_{30-90}$ & $15-29$ & $10-1,000$ & 12 \\
\hline
\end{tabular}

extent of the inter-facility difference in sensitivity and reliability of this new testing method. The concentrations of E-4031 that caused early afterdepolarization and/or triggered activity were close to each other among the 3 facilities; however, there were some variations in the basal absolute values of inter-spike interval and FPDcF. Since we used the same batch of the cardiomyocytes, these differences might be induced by small inter-facility variability in the net culture period, the cell density on recording electrodes and/or experimental temperature. It should be noted that there was no difference in inter-spike interval or FPDcF among the 3 facilities when compared using percentage change.

In conclusion, we demonstrated that the use of the standardized protocol for the iPS cell-derived cardiomyocyte sheets can minimize inter-facility difference in detecting the drug-induced repolarization delay and arrhythmia. While further studies are needed to establish the currently proposed protocol including the assessment of variability across batches, more reference compounds and clinical predictability, information described in this paper may help predict the potential of the drug-induced repolarization delay and arrhythmias with this new technology. Also, methodological work for high-throughput evaluation is now ongoing.

\section{Acknowledgments}

This study was supported in part by the Research Promotion Grant from Toho University Graduate School of Medicine (No. 13-01) and a Regulatory Science Research Grant from the Ministry of Health Labour and Welfare. The authors thank Ms. Misako Nakatani, Ms. Tomoko Ohnishi, and Dr. Mitsuyoshi Luke Saito for their technical assistance and Alpha MED Scientific, Inc. and iPS Academia Japan, Inc. for their technical advice.

\section{Conflicts of Interest}

The authors declare no conflicts of interest.

\section{References}

1 ICH Harmonised Tripartite Guideline. The Non-clinical Evaluation of the Potential for Delayed Ventricular Repolarization (QT Interval Prolongation) by Human Pharmaceuticals S7B. Recommended for adoption at step 4 of the ICH process on 12 May 2005 by the ICH Steering Committee. ICH; (http://www. ich.org/fileadmin/Public_Web_Site/ICH_Products/Guidelines/ Safety/S7B/Step4/S7B_Guideline.pdf)

2 ICH Harmonised Tripartite Guideline. The Clinical Evaluation of QT/QTc Interval Prolongation and Proarrhythmic Potential for Non-Antiarrhythmic Drugs E14. Recommended for adoption at step 4 of the ICH process on 12 May 2005 by the ICH Steering Committee. ICH; (http://www.ich.org/fileadmin/Public_Web_Site/ 
ICH_Products/Guidelines/Efficacy/E14/E14_Guideline.pdf)

3 Darpo B. The thorough QT study four years after the implementation of the ICH E14 guidance. Br J Pharmacol. 2010;159: 49-57.

4 E14 Implementation Working Group ICH E14 Guideline: The Clinical Evaluation of QT/QTc Interval Prolongation and Proarrhythmic Potential for Non-Antiarrhythmic Drugs Questions \& Answers (R1). 2012. (http://www.ich.org/fileadmin/ Public_Web_Site/ICH_Products/Guidelines/Efficacy/E14/ E14_Q_As_R1_step4.pdf)

5 Sugiyama A, Hashimoto H, Nakamura Y, Fujita T, Kumagai Y. QT/QTc study conducted in Japanese adult healthy subjects: a novel xanthine oxidase inhibitor topiroxostat was not associated with QT prolongation. J Clin Pharmacol. In press.

6 Giorgi MA, Bolaños R, Gonzalez CD, Di Girolamo G. QT interval prolongation: preclinical and clinical testing arrhythmogenesis in drugs and regulatory implications. Curr Drug Saf. 2010;5:54-57.

7 Chi KR. Revolution drawing in cardiotoxicity testing. Nat Rev Drug Discov. 2013;12:565-567.

8 Yamazaki K, Hihara T, Taniguchi T, Kohmura N, Yoshinaga T, Ito $\mathrm{M}$, et al. A novel method of selecting human embryonic stem cell-derived cardiomyocyte clusters for assessment of potential to influence QT interval. Toxicol In Vitro. 2012;26:335-342.

9 He JQ, Ma Y, Lee Y, Thomson JA, Kamp TJ. Human embryonic stem cells develop into multiple types of cardiac myocytes: action potential characterization. Circ Res. 2003;93:32-39.

10 Itzhaki I, Maizels L, Huber I, Zwi-Dantsis L, Caspi O, Winterstern A, et al. Modelling the long QT syndrome with induced pluripotent stem cells. Nature. 2011;471:225-229.

11 Nalos L, Varkevisser R, Jonsson MK, Houtman MJ, Beekman $J D$, van der Nagel $R$, et al. Comparison of the $I_{K r}$ blockers moxifloxacin, dofetilide and E-4031 in five screening models of pro-arrhythmia reveals lack of specificity of isolated cardiomyocytes. Br J Pharmacol. 2012;165:467-478.

12 Schaaf S, Shibamiya A, Mewe M, Eder A, Stöhr A, Hirt MN, et al. Human engineered heart tissue as a versatile tool in basic research and preclinical toxicology. PLoS One. 2011;6:e26397.

13 Tanaka T, Tohyama S, Murata M, Nomura F, Kaneko T, Chen H, et al. In vitro pharmacologic testing using human induced pluripotent stem cell-derived cardiomyocytes. Biochem Biophys Res Commun. 2009;385:497-502.

14 Fridericia LS. [Die sytolendauer in elektrokardiogramm bei normalen menshen und bei herzfranken]. Acta Med Scand. 1920;53:469-486. (text in German)

15 Fujiki A, Tani M, Mizumaki K, Shimono M, Inoue H. Electro- physiologic effects of intravenous E-4031, a novel class III antiarrhythmic agent, in patients with supraventricular tachyarrhythmias. J Cardiovasc Pharmacol. 1994;23:374-378.

16 Verheijck EE, van Ginneken AC, Bourier J, Bouman LN. Effects of delayed rectifier current blockade by E-4031 on impulse generation in single sinoatrial nodal myocytes of the rabbit. Circ Res. 1995; 76:607-615.

17 Brouillette J, Lupien MA, St-Michel C, Fiset C. Characterization of ventricular repolarization in male and female guinea pigs. $\mathrm{J}$ Mol Cell Cardiol. 2007;42:357-366.

18 Tabo M, Komatsu R, Isobe T, Honda M, Yamada Y, Kimura K. Accurate detection of drug-induced delayed ventricular repolarization with a suitable correction formula in Langendorff guinea pig heart. J Toxicol Sci. 2010;35:687-698.

19 Asano Y, Davidenko JM, Baxter WT, Gray RA, Jalife J. Optical mapping of drug-induced polymorphic arrhythmias and torsade de pointes in the isolated rabbit heart. J Am Coll Cardiol. 1997;29:831-842.

20 Maruyama M, Lin SF, Xie Y, Chua SK, Joung B, Han S, et al. Genesis of phase 3 early afterdepolarizations and triggered activity in acquired long-QT syndrome. Circ Arrhythm Electrophysiol. 2011;4:103-111.

21 Zhou Z, Gong Q, Ye B, Fan Z, Makielski JC, Robertson GA, et al. Properties of HERG channels stably expressed in HEK 293 cells studied at physiological temperature. Biophys J. 1998;74: 230-241.

22 Sanguinetti MC, Jurkiewicz NK, Scott A, Siegl PK. Isoproterenol antagonizes prolongation of refractory period by the class III antiarrhythmic agent E-4031 in guinea pig myocytes. Mechanism of action. Circ Res. 1991;68:77-84.

23 Wettwer E, Scholtysik G, Schaad A, Himmel H, Ravens U. Effects of the new class III antiarrhythmic drug E-4031 on myocardial contractility and electrophysiological parameters. J Cardiovasc Pharmacol. 1991; 17:480-487.

24 Omata T, Kasai C, Hashimoto M, Hombo T, Yamamoto K. QT PRODACT: comparison of non-clinical studies for drug-induced delay in ventricular repolarization and their role in safety evaluation in humans. J Pharmacol Sci. 2005;99:531-541.

25 Hashimoto K, Haruno A, Matsuzaki T, Hirasawa A, Awaji T, Uemura Y. Effects of a new class III antiarrhythmic drug (E-4031) on canine ventricular arrhythmia models. Asia Pac J Pharmacol. 1991;6:127-137.

26 Katritsis D, Morgan J, Brachmann J, Bygrave A, O’Farrell D, Rowland E, et al. Electrophysiological effects of E 4031, a drug with selective class III properties, in man. Pacing Clin Electrophysiol. 1997;20:930-937. 